\title{
Evaluación de tres alternativas para el control de varroasis (Varroa destructor) en apiarios ecuatorianos
}

Evaluation of three alternatives for the control of varroasis (Varroa destructor) in ecuadorian apiaries

Fecha de recepción: 11 de agosto de 2018 Fecha de aprobación: 17 de octubre de 2018

\author{
Byron Díaz-Monroy ${ }^{1}$ \\ Juan Moyón-Moyón ${ }^{2}$ \\ María Fernanda Baquero-Tapia ${ }^{3}$
}

\section{Resumen}

En Chimborazo, Ecuador, se evaluó tres tratamientos para el control de Varroasis (Varroa destructor) en abejas, T1: Ácido Fórmico (al 85\%), T2: Ácido Oxálico (al 10\% en jarabe de azúcar), T3: Timol (al 99\% diluido en aceite de oliva), y un tratamiento control (T0), con un diseño completamente al azar y cuatro réplicas (colmenas) por cada tratamiento, durante 30 días de aplicación de los tratamientos sobre las colmenas tipo Langstroth. Se realizó un estudio diagnóstico inicial para determinar el nivel de infestación con varroasis y seleccionar las 16 colmenas con igual nivel de infestación, luego se aplicó los tratamientos, así el ácido fórmico se aplicó dos veces, una cada 15 días con un rociador, el ácido oxálico se aplicó cuatro veces, una cada 4 días en solución azucarada, el timol se aplicó dos veces, una cada 8 días con rociador y diluido en aceite de oliva. Se determinó una mejor eficacia $(95,1 \%)$ en el control de varroasis con el Ácido fórmico, sin afectar la población de abejas, tanto por el modo de acción selectivo del producto en la fase reproductiva y forética de las varroas, como por su largo periodo de acción, además de su bajo costo (8,2 dólares/colmena), por lo que se recomienda utilizar este producto para el control de Varroa destructor en Apis mellifera.

Palabras clave: abejas; acido fórmico; ácido oxálico; colmena; timol; varroasis.

\section{Abstract}

In Chimborazo, Ecuador, three treatments were evaluated for the control of Varroasis (Varroa destructor) in bees, T1: Formic Acid (at 85\%), T2: Oxalic Acid (at 10\% in sugar syrup), T3: Thymol (at 99\% diluted in olive oil), and a control treatment (T0), with a

\footnotetext{
${ }^{1}$ Ph. D. Escuela Superior Politécnica de Chimborazo (Chimborazo, Ecuador). bdiaz@espoch.edu.ec. ORCID: $\underline{0000-}$ 0003-3721-7994.

${ }^{2}$ Escuela Superior Politécnica de Chimborazo (Chimborazo, Ecuador). imoyon@espoch.edu.ec.

${ }^{3}$ M. Sc. Universidad Nacional de Chimborazo (Chimborazo, Ecuador). mbaquero@unach.edu.ec. ORCID: 0000-00033331-528X. 
completely randomized design and four replicas (hives) for each treatment, during 30 days of application of the treatments on Langstroth hives. An initial diagnostic study was carried out to determine the level of varroasis infestation and select the 16 hives with the same level of infestation, then the treatments were applied, so the formic acid was applied twice, once every 15 days with a sprayer, the acid oxalic was applied four times, one every 4 days in sugar solution, thymol was applied twice, once every 8 days with spray and diluted in olive oil. A better efficacy (95.1\%) was determined in the control of varroasis with formic acid, without affecting the population of bees, both by the mode of selective action of the product in the reproductive and foretic phase of the varroas, as by its long period of action, in addition to its low cost ( 8.2 dollars/hive), so it is recommended to use this product for the control of Varroa destructor in Apis mellifera.

Keywords: bees; formic acid; hive; oxalic acid; thymol; varroasis.

\section{Para citar este artículo:}

Díaz-Monroy, B., Moyón-Moyón, J., \& Baquero-Tapia, M. F. (2019). Evaluación de tres alternativas para el control de varroasis (Varroa destructor) en apiarios ecuatorianos. Ciencia $\quad y \quad$ Agricultura, 16(1), 63-78. DOI: https://doi.org/10.19053/01228420.v16.n1.2019.8832.

Esta obra está bajo licencia internacional Creative Commons Reconocimiento 4.0 


\section{Introducción}

Apicultura, proviene del latín Apis (abeja) y Cultura (cultivo), es decir, la ciencia que se dedica al cultivo o cría de las abejas utilizando la tecnología, para obtener beneficios económicos, es el arte y la ciencia de la cría y mantenimiento de las abejas con vistas a obtener de su trabajo, los productos apícolas y generar un beneficio económico (Sánchez, 2003).

En Ecuador y el mundo, la apicultura es una técnica de muy larga data, la cual experimentó importantes avances científicos a partir del siglo pasado, sin embargo, en la actualidad su nivel de explotación es insuficiente, dado que no ha existido una adecuada masificación del conocimiento tecnológico en este rubro, siendo evidente una falta de personal calificado para un correcto manejo de las explotaciones, las que logran niveles de producción muy lejanos a la potencialidad de nuestra flora (Jordán y Lalangui, 2016). La mayor población de colmenas, se encuentran en los Andes Ecuatorianos, su principal floración es el Eucaliptus glóbulus, la abeja africanizada, ingresada al país en los años 70 , es la más común. A pesar de su amplia biodiversidad, el Ecuador no ha logrado repuntar su producción melífera, tal es así que este sector apenas mueve el 0,1 \% de la economía nacional, con una producción anual promedio por colmena de $35 \mathrm{~kg}$, numero promedio de colmenas por apicultor 25, cosechas al año 3 a 4, producción anual mínima por colmena 12 kg, producción anual máxima por colmena 90 kg (Cabrera, 2007).

La apicultura en la actualidad enfrenta una seria problemática sanitaria, las infestaciones parasitarias causadas por el ácaro Varroa destructor anteriormente Varroa jacobsoni Oudeman, que está distribuido en la mayor parte del mundo y es considerada una de las peores plagas de las abejas (Ball, \& Alderson, 2008 citados por Ruíz et al., 2012), pues afectan a la abeja melífera en todos sus estadios de desarrollo (crías y adultas), es conocido que este ácaro actúa también de factor predisponente para que se desarrollen otras infecciones secundarias: virosis ascosferosis y loques, puesto que este parasito perfora y succiona la hemolinfa de la cría y abejas adultas debilitándolas y propagando enfermedades infecciosas, colonias de abejas infestadas que al no 
ser tratadas mueren o se produce un abandono de la colmena y del apiario, dejando como consecuencia principal el descenso en la polinización de las plantas, así como también la disminución de la producción de miel, polen y otros productos apícolas. El daño que la varroosis causa depende del grado de infestación de las colonias afectadas. Informes científicos estiman que el efecto negativo sobre la productividad comienza cuando la población de ácaros alcanza $10 \%$ de las abejas adultas en una colonia; en este sentido, cuando la infestación llega a ser de 30\% a 40\%, normalmente termina con la colonia (De Jong, 1997, citado por Espinosa y Guzmán, 2007).

El desarrollo de la apicultura en Ecuador se ha visto afectado por la poca o nula investigación en este insecto benéfico, en relación a la patología causada por la varroasis, sus efectos y control eficaz principalmente. Los esfuerzos para controlar la varroasis se han enfocado al uso de acaricidas sintéticos, varios de éstos proveen alto grado de control del ácaro. Sin embargo, estos acaricidas tienen serios inconvenientes; pueden promover el desarrollo de resistencia a su principio activo en los ácaros, son tóxicos para las abejas y dejan residuos químicos en la miel para consumo humano (Milani, 1999). Un acercamiento de corto plazo al control de la varroasis es el uso de algunos acaricidas naturales, ya que tienen baja toxicidad y bajo impacto ambiental, porque no dejan residuos en la miel, o porque sus residuos se degradan o volatilizan en poco tiempo (Espinosa y Guzmán, 2007). Pocos productos naturales han mostrado eficacia contra Varroa, entre ellos están el ácido fórmico y el aceite esencial de timol (Stanghellini, 2004). La utilización de productos orgánicos en el control de varroasis como el ácido fórmico, ácido oxálico y timol en diferentes lugares del mundo han dado buenos resultados en diferentes épocas de aplicación, diferentes concentraciones, dosis y frecuencia de aplicación, el estudio del modo de acción de estos productos, así como su selectividad sobre las varroas, son parámetros que deben ser considerados en una investigación para obtener resultados satisfactorios.

La presente investigación determinó la pertinencia, eficacia y costo de los tratamientos de la enfermedad en base a productos naturales, para ello se consideraron como objetivos el realizar un diagnóstico inicial para determinar la 
presencia e incidencia de Varroa destructor en tres apiarios de la provincia de Chimborazo, Ecuador, luego aplicar y evaluar la eficacia de tres tratamientos en base a Ácido fórmico, Ácido Oxálico y Timol, en las colmenas infestadas con Varroa destructor y finalmente determinar el costo de estas alternativas sanitarias.

\section{Materiales y métodos}

La metodología utilizada para cumplir con los objetivos de la investigación, se detalla en los siguientes acápites:

\section{A. Localización y duración del experimento}

La investigación tuvo dos fases, una de diagnóstico, realizándose en la comunidad Asactus, perteneciente al Cantón Chambo y en las comunidades Socorro y Nitiluiza pertenecientes al cantón Riobamba, cantones pertenecientes a la provincia de Chimborazo, mientras que la fase de aplicación y evaluación de los tratamientos se realizó únicamente en la comunidad Asactus, Cantón Chambo, Provincia de Chimborazo. El trabajo completo tuvo una duración de 120 días.

\section{B. Unidades experimentales}

Para la investigación se utilizó 16 colmenas tipo Langstroth, conformadas por una alza completa más una media alza, a las cuales se aplicó 4 tratamientos (Acido fórmico al 85\%, Acido oxálico al 10\%, timol al 99\% diluido en aceite de oliva y un testigo), a cada tratamiento se asignó 4 colmenas (réplicas) dando un total de 16 unidades experimentales (Colmenas).

\section{Tratamiento y diseño experimental}

Los cuatro tratamientos evaluados en este trabajo, así como el diseño experimental utilizado, fueron los siguientes:

- T1= Acido fórmico al 85\%, con dos aplicaciones una cada 15 días (120 ml en cada aplicación/ colmena).

- $\mathrm{T} 2$ = Acido oxálico al 10\%, en una solución compuesta por 1:10:10, para acido Oxálico, azúcar y agua, respectivamente, 4 aplicaciones con intervalo de 4 días, entre cada una, con dosis de $5 \mathrm{ml} /$ bastidor. 
- T3 = Timol al 99.9\%, disuelto en aceite de oliva, dos aplicaciones con intervalo de 8 días, entre cada una, $10 \mathrm{~g} /$ colmena.

- T0 = Testigo, sin ningún producto

Las colmenas utilizadas fueron homogéneas en cuanto al nivel de infestación por Varroa, por lo que se distribuyeron bajo un Diseño Completamente al Azar.

\section{Análisis estadístico}

Los resultados obtenidos fueron analizados con sistema estadístico SAS 8.2, además se aplicaron los siguientes análisis estadísticos:

- Análisis de Varianza (ADEVA)

- Separación de medias según Tukey al 0.05 y 0.01 de significancia

\section{E. Procedimiento experimental}

El diagnóstico inicial se realizó en tres apiarios de la provincia de Chimborazo mediante la determinación de caída de ácaros (varroas) sobre una charola revestida con vaselina durante un día, para luego proceder a determinar el porcentaje de incidencia mediante la prueba de David De Jong (1997), obteniéndose así las 16 colmenas con infestaciones similares para ser sometidas a los tratamientos correspondientes.

En la fase de aplicación de los tratamientos se procedió a la toma de datos de variables poblacionales pre-aplicación de los tratamientos 7 días antes, la aplicación de los tratamientos tuvo una duración de 30 días, con tratamientos de corta duración para ácido oxálico y timol, mientras que para ácido fórmico se utilizó un tratamiento de larga duración, después del día treinta, se procedió a quitar todos los residuos de los productos en estudio de las colmenas, la toma de datos finales de variables poblacionales como: varroas caídas, \% de infestación, peso de colmena, ritmo de postura, población de abejas, fueron tomados a los 75 días después de la última aplicación de los tratamientos sobre las colmenas, mientras que el porcentaje de incidencia de varroa durante la aplicación de los tratamientos fue tomado a los 15 días después de la aplicación de los tratamientos, la mortalidad de abejas adultas y cría se evaluó durante los 30 días de aplicación de los tratamientos. 


\section{Resultados y discusión}

Los resultados obtenidos al final del trabajo, se presentan en secciones de acuerdo a los objetivos planteados y cumplidos:

\section{A. Evaluación de la eficiencia de tres alternativas para el control de varroasis (Varroa destructor)}

\section{1) Número de Varroas caídas $/ \mathrm{dm}^{2} / \mathrm{día}$ pre y pos- aplicación del} tratamiento: El número de varroas caídas $/ \mathrm{dm}^{2} /$ día pre-aplicación de los tratamientos, fue de 5,$04 ; 4,96 ; 5,02$ y 5,03 para las colmenas pertenecientes a los tratamientos Testigo, Acido Fórmico, Acido Oxálico y Timol, respectivamente, alcanzado un promedio general de 5,01 varroas caídas/dm²/día, lo que significó una población alta de varroas en las colmenas antes de la aplicación de los tratamientos, como se aprecia en la Tabla 1.

La caída de varroas pos-aplicación de los tratamientos registraron diferencias altamente significativas $(P<0.01)$, de esta manera en las colmenas sometidas al tratamiento de ácido fórmico presentaron menor promedio de caída de varroas/dm²/día con 0,14 varroas, seguido por el promedio obtenido en las colmenas tratadas con ácido oxálico con 0,6 varroas, posteriormente se ubicó el promedio determinado en las colmenas en las cuales se aplicó el tratamiento de timol, alcanzando un promedio de 1,08 varroas, finalmente el grupo testigo con 5,53 varroas/dm²/día, como se ven la Tabla 2 .

Tabla 1. Estudio de incidencia de varroasis (Varroa destructor) y variables poblacionales de abejas.

\begin{tabular}{|l|c|c|c|c|c|c|}
\hline \multirow{2}{*}{\multicolumn{1}{c|}{ Indicador }} & \multicolumn{3}{c|}{ Tratamientos } & \multirow{2}{*}{$\begin{array}{c}\text { Error } \\
\text { estándar }\end{array}$} \\
\cline { 2 - 7 } & Testigo & Acido Fórmico & Acido oxálico & Timol & $\overline{\mathbf{X}}$ & Án \\
\hline № Varroas caídas/dm/día pre-tratamiento & 5,0 & 4,96 & 5,02 & 5,03 & 5,0 & 0,09 \\
\hline Infestación Varroas pre-tratamiento (\%) & 10,83 & 10,50 & 11,28 & 10,75 & 10,84 & 0,27 \\
\hline Pesos inicial colmena (kg) & 10,25 & 10,05 & 9,85 & 10,38 & 10,13 & 0,22 \\
\hline Ritmo de postura inicial de la reina(№) & 1312,5 & 1375 & 1312,5 & 1437,5 & $1.359,4$ & 65,05 \\
\hline Población inicial de abejas. (№) & 20000 & 20000 & 20000 & 20000 & 20000 & 0,000 \\
\hline
\end{tabular}


Tabla 2. Evaluación de la eficiencia de tres alternativas para el control de varroa

(Varroa destructor).

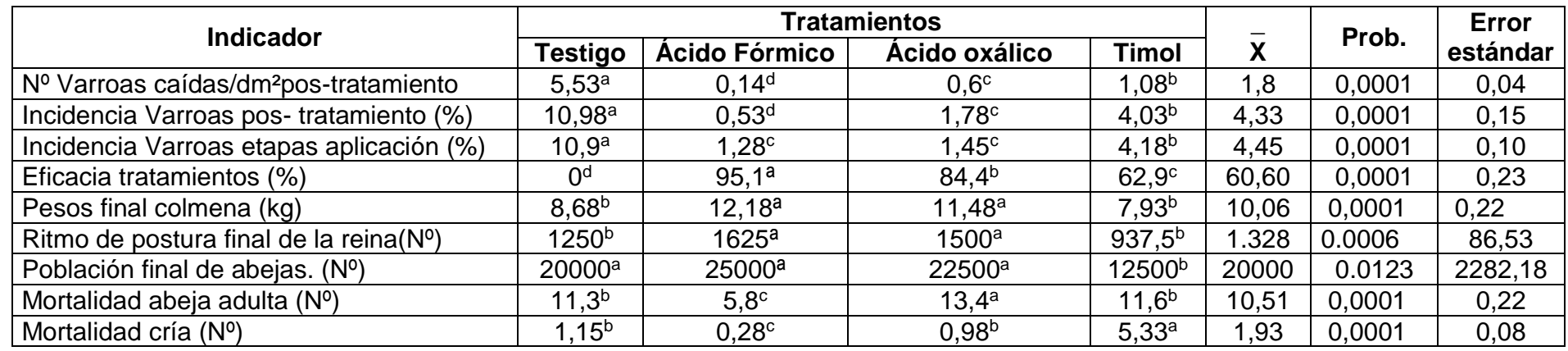

Letras iguales no difieren estadísticamente de acuerdo a Tukey $(\mathrm{P} \leq 0.05$ y $\mathrm{P} \leq 0.01)$; Prob: Probabilidad; ns: Diferencia no significativa entre promedios. ${ }^{* \star}$ Diferencia altamente significativa entre medias de los tratamientos.

Los resultados obtenidos demuestran que el ácido fórmico al $85 \%$ bajó en mayor proporción la población de varroas esto debido a que el producto actuó tanto en la fase forética como en la fase reproductiva de la varroa, a través de la evaporación del producto, afectando así el sistema respiratorio de la varroa lo cual es corroborado por Eguaras (2003), quien manifiesta que en el interior de las colmenas, el ácido actúa por evaporación, afectando el sistema respiratorio de los ácaros que se encuentran sobre la abeja adulta como a los que están en fase reproductiva dentro de las celdas de cría.

\section{2) Porcentaje de Infestación de varroa pre y pos-aplicación del}

tratamiento: El porcentaje de infestación de varroas pre-aplicación de los tratamientos, al inicio del presente estudio fue de 10,83; 10,5; 11,28 y 10,75\% para las colmenas que fueron sometidas al control de varroas mediante los tratamientos Testigo, Acido Fórmico, Acido Oxálico y Timol, respectivamente, alcanzado un promedio general de 10,84; lo cual demuestra que las colmenas en ese momento tenían una infestación natural alta de Varroa destructor, como se observa en la Tabla 1.

El porcentaje de infestación por Varroa destructor pos-aplicación de los tratamientos registraron diferencias altamente significativas $(P<0.01)$, de esta manera el tratamiento Acido Fórmico presentó menor promedio de infestación de varroa con $0,525 \%$ seguido por el tratamiento acido oxálico aplicado directamente sobre las abejas adultas en dosis de $5 \mathrm{ml} /$ marco lleno de abejas 
presentando un promedio de infestación de 1,775\%, posteriormente se ubicó el tratamiento timol en dosis de $10 \mathrm{~g}$ de timol diluido en $17 \mathrm{ml}$ de aceite de oliva, alcanzando un promedio de infestación de 4,075\%, y finalmente el testigo que tuvo una infestación promedio de $10,975 \%$, como lo podemos observar en la Tabla 2.

\section{3) Porcentaje de infestación de varroa durante la aplicación de los}

tratamientos: Este valor se tomó 15 días después de las primeras aplicaciones de las tres alternativas para el control de varroa, registrándose diferencias altamente significativas $(P<0.01)$, de esta manera el tratamiento acido fórmico y acido oxálico presentaron los menores promedios de infestación de varroa en la colmena con $1,28 \%$ y $1,45 \%$, posteriormente se ubicó el tratamiento timol alcanzando un promedio de 4,18 \%, finalmente el testigo tuvo un promedio de $10,90 \%$ de infestación de varroa en la colmena, como se observa en la Tabla 2.

De acuerdo a los datos tomados a los 75 días después de la última aplicación de los tratamientos, se evidencia que el tratamiento con ácido fórmico con dos aplicaciones disminuyó 9,23 puntos porcentuales de infestación de varroa, mientras que para el ácido oxálico en el mismo tiempo pero con 4 aplicaciones del producto redujo 9,83 puntos porcentuales de infestación de varroa y para timol en el mismo tiempo con dos aplicaciones del producto redujo 6,575 puntos porcentuales de infestación de varroa y finalmente para el tratamiento testigo no se observó ninguna disminución porcentual de infestación, más bien hubo un incremento de 0,07 puntos porcentuales de infestación de Varroa destructor en el mismo tiempo que fueron evaluados los demás tratamientos.

4) Eficacia de los tratamientos: El porcentaje de eficacia de las tres alternativas para el control de Varroa destructor registró diferencias altamente significativas $(\mathrm{P}<0.01)$, de esta manera el tratamiento con ácido fórmico con dos aplicaciones cada 15 días presentó la mejor eficacia en el control de varroa destructor en la colmena con un promedio de 95,1\% de eficacia, seguido por el tratamiento acido oxálico al $10 \%$ con cuatro aplicaciones cada 4 días obteniendo un promedio de $84,45 \%$, posteriormente se ubicó el tratamiento 
timol al 99\% diluido el aceite de oliva con dos aplicaciones cada 8 días alcanzando un promedio de $62,8 \%$, finalmente el testigo que no tuvo eficacia en el control de varroa destructor, como se observa en la Tabla 2.

Eguaras (2003), encontró que con tratamientos de larga duración se lograron valores de eficacia superiores al $90 \%$ con rangos de variabilidad de $76,7 \%$ a 99\%. Mientras que Arculeo (2000), citado por Silva (2006), reporta $82.8 \%$ de eficacia en colmenas con cría, para la aplicación de ácido oxálico al 10\% en solución azucarada 1:1, mientras que para colmenas sin cría reporta eficacias de $94,1 \%$. Por su parte, Llorete et al (1996) reportó que mediante la aplicación de $10 \mathrm{~g}$ de timol pulverizado y homogenizado sobre papel aluminio abierto y colocado sobre la parte superior de los cuadros, con cuatro aplicaciones con intervalo de 7 días, obtuvo una eficacia del 99,2\%.

\section{B. Peso de colmenas pre y pos - aplicación de los tratamientos}

El peso de las colmenas pre-aplicación de los tratamientos, al inicio del estudio fue 10,$25 ; 10,05 ; 9,85$ y 10,38 $\mathrm{Kg}$ para las colmenas que fueron sometidas al control de varroas mediante los tratamientos Testigo, Acido Fórmico, Acido Oxálico y Timol respectivamente, alcanzado un promedio general de $10,13 \mathrm{Kg}$ y disponiéndose de unidades experimentales homogéneas al iniciar el experimento, como se observa en Tabla 1.

El peso de las colmenas pos-aplicación de los tratamientos registró diferencias altamente significativas $(P<0.01)$, de esta manera el tratamiento acido fórmico y acido oxálico presentaron mayores pesos con promedios de 12,18 y 11,48 Kg respectivamente, seguido por el tratamiento con timol y el testigo, con pesos promedio de 7,93 y 8,68 $\mathrm{Kg}$ respectivamente, como se observa en Tabla 2.

La disminución del peso en las colmenas del tratamiento testigo está relacionado a la mortalidad de la cría o a la expulsión de abejas con mal formación en el cuerpo causado por las varroas lo cual daría una disminución de la población de abejas adultas trayendo como consecuencia un bajo nivel de pecoreo, el resultado sería una pérdida gradual del peso de la colmena además porque el consumo interno de miel y polen es mayor a la recolectada por las 
abejas adultas, esto porque las crías que fueron infestadas con varroas serán expulsadas de la colmenas no logrando llegar a la etapa adulta para el pecoreo, la disminución de peso en las colmenas tratadas con timol se debe a que el timol influye sobre un comportamiento higiénico de las abejas ya que eliminan todas las crías lo cual trae una disminución de la población de abejas en la colmena solo durante el proceso del tratamiento ya que después de los tratamientos regresaría a la dinámica normal de la colmena, por lo cual se recomendaría hacer este tratamiento solo en colmenas fuertes.

\section{Mortalidad de abeja adulta}

La mortalidad de abejas durante la aplicación de los tratamientos registro diferencia altamente significativas $(P<0.01)$, de esta manera el tratamiento acido fórmico presentó un menor promedio muertes de abejas/día con 5,78 seguido por el testigo y timol, con promedios de 11,30 y 11,57 abejas muerta/día respectivamente y finalmente el tratamiento ácido Oxálico al 10\% quien tu tubo un mayor promedio de mortalidad de abejas/día con 13,38 como se observa en Tabla 2.

La mortalidad de abejas en la presente investigación para acidó fórmico fue menor debido a que el producto fue selectivo ya que hubo una mayor mortalidad de ácaros sin afectar a las abejas lo cual es corroborado por Eguaras (2003), quien manifiesta que se debe a la mayor inhibición de la respiración en los ácaros en comparación con las abejas, como también por la menor capacidad metabólica y de regulación, mientras que para ácido oxálico en nuestra investigación hubo una mayor mortalidad de abejas lo cual es semejante a lo que reporta Silva (2006), quien sostiene que las tres concentraciones del ácido: 5, 10 y 20\%, provocan una notable disminución de la población de abejas dentro de la colmena, lo cual se deba a una muerte por asfixia ya que al momento de aplicar el ácido oxálico en jarabe de azúcar por medio de goteo este producto haya obstaculizado las vías respiratorias de las abejas, como lo manifiestan Charriere e Imdorf (2001), citados por Silva (2006), quienes encontraron que las abejas no ingieren la solución, es más, el azúcar mejoraría la adherencia del producto, incrementando la efectividad de éste, mientras que para timol la mortalidad fue casi igual a la del testigo lo cual nos 
manifiestas que no afecto sobre las abejas adultas ya que la mortalidad pueda estar determinada a la apoca de floración en donde a mayor actividad de pecoreo la mortalidad aumenta y viceversa.

\section{Mortalidad de cría}

La mortalidad de cría de abeja en estado de pupa durante las aplicaciones de los tratamientos registro diferencia altamente significativas $(P<0.01)$, de esta manera el tratamiento acido fórmico al $85 \%$ presento un menor promedio de muerte de cría/día con 0,275, seguido por el tratamientos ácido Oxálico y testigo quienes tuvieron un promedio de mortalidad de cría/día de 0,975 y 1,15 respectivamente finalmente el tratamiento timol quien obtuvo un mayor promedio de mortalidad de cría/día con 5,32, como se ven en la Tabla 2.

La mortalidad de cría en la investigación para ácido fórmico, acido oxálico y testigo fueron menores debido a los productos no entran en contacto directo con la cría como lo manifiesta Eguaras, et al. (2000), quien manifiesta que el ácido actúa por evaporación alcanzando tanto a los ácaros que se encuentran sobre la abeja adulta como a los que están en fase reproductiva dentro de las celdas de cría, mientras que para acido oxálico su aplicación es directa sobre las abejas ya que el ácido no actúa por evaporación sino por contacto directo lo cual lo plantea Nanetti (2007), y que la presencia de azúcar en la solución reviste un papel importante como soporte, favoreciendo la acción del principio activo. Así mismo el timol no entra en contacto con la cría, pero presenta alta mortalidad la cual se deba a que el timol un estímulo excesivo de comportamiento higiénico sacando mucha cría según Ruíz (2012), lo cual indicaría las diferencias estadísticas entre los tratamientos.

\section{E. Costos de la tecnología de aplicación de los tratamientos}

Para la evaluación de costos de la tecnología se consideraron los costos de las aplicaciones/tratamiento sumado a ello el costo de mano de obra y de transporte, el costo total de materiales, mano de obra y transporte están divididos para 4 colmenas ya que en el proceso de investigación los materiales, la mano de obra y transporte se utilizaba en 4 colmenas en todos los tratamientos, dando como resultado un menor costo/colmena tratada para el 
tratamiento con ácido fórmico al 85\% con dos aplicaciones cada 15 días de 8,2 dólares/colmena tratada, seguido por el ácido oxálico al $10 \%$ con 4 aplicaciones con intervalo de cuatro días con un costos/colmena tratada de 13,2 dólares, posteriormente el tratamiento timol al 99\% diluido en aceite de oliva con dos aplicaciones cada 8 días con un costo/colmena tratada de 18,9 dólares y finalmente el testigo con un costo de 5,89 dólares, costo que toma en cuenta solo el costo de la mano de obra y del transporte para las dos revisiones que duró el proceso investigativo pero al mismo tiempo no se observó una reducción del porcentaje de infestación y más bien se notó un leve incremento, como se observa en la Tabla 3.

Tabla 3. Evaluación de costos de la tecnología de aplicación de las tres alternativas de tratamiento para el control de varroasis (Varroa destructor).

\begin{tabular}{|l|c|c|c|c|}
\hline \multirow{2}{*}{\multicolumn{1}{|c|}{ Concepto }} & \multicolumn{4}{c|}{ Tratamientos } \\
\cline { 2 - 5 } & Testigo & Ácido Fórmico & Ácido Oxálico & Timol \\
\hline Aplicación tratamiento & 0,00 & 2,30 & 1,43 & 13,01 \\
\hline Transporte & 2,00 & 2,00 & 4,00 & 2,00 \\
\hline Mano de obra & 3,89 & 3,89 & 7,78 & 3,89 \\
\hline Costo total en dólares & 5,89 & 8,20 & 13,20 & 18,90 \\
\hline
\end{tabular}

\section{Conclusiones}

El diagnóstico mediante la prueba de David De Jong determinó que el apiario de la comunidad Asactus, cantón Chambo, el 87,5\% de las colmenas tienen infestaciones mayores al $10 \%$ de varroasis, mientras que en los apiarios ubicados del cantón Riobamba, comunidad Socorro, el 62,5\% de las colmenas tiene infestaciones iguales o mayores al $10 \%$ y en la comunidad Palacio Real el $100 \%$ de las colmenas tienen una infestación promedio de 4,63\%.

Luego de la aplicación de los tratamientos, se determinó que la utilización del Ácido Fórmico al 85\% fue el mejor, alcanzando una eficacia promedio del $95,1 \%$ para el control de Varroa destructor, sin afectar a la población de abejas de la colmena, mientras que el tratamiento con ácido oxálico al 10\% alcanzó una eficacia promedio de $84,45 \%$, influyendo negativamente sobre las abejas 
adultas, y para el tratamiento timol al 99\% diluido en aceite de oliva se obtuvo una eficacia promedio de $62,8 \%$ incidiendo negativamente con mortalidad de la cría.

El costo/colmena para el control de Varroasis (Varroa destructor) es menor al utilizar acido fórmico con un valor de 8,2 USD/colmena tratada, mientras que se incurre en mayores costos al utilizar Acido oxálico y Timol con montos de 13,2 y 18,9 USD/ colmena tratada, respectivamente.

\section{Recomendaciones}

Utilizar ácido fórmico al 85\% en dos aplicaciones cada 15 días para el control de Varroa destructor, ya que a más de ser inofensivo para las abejas presenta la mayor eficacia y bajo costo.

Difundir los resultados obtenidos a nivel de apicultores de la región Sierra Centro del país con el fin de controlar la parasitosis de Varroa destructor en abejas.

Realizar otras investigaciones en donde se evalúen, concentraciones menores a las utilizadas en la presente investigación, con el fin de reducir los costos del tratamiento de esta enfermedad parasitaria.

\section{Referencias}

Ball, B., \& Alderson, P. (2008). Immunolocalization of deformed wing virus particles within the mite Varroa destructor. Journal of General Virology, 89, 1685-1689. DOI: https://doi.org/10.1099/vir.0.83223-0.

Bulacio, N.; Basualdo, M. y Eguaras, M. (2010). Actividad Varroocida del timol en colonias de Apis mellifera $L$. de la provincia de Santa Fe. Revista de la Sociedad Entomológica Argentina, $12(1), 1668-3498$.

Cabrera, J. (2007). Perspectivas de la Apiterapia en el Ecuador. Quito, Ecuador: Carisma.

De Jong, D. (1997). Mites: Varroa and other parasites of brood. Honey bee pests, predators and diseases. Ithaca New York: Root Publishing.

Eguaras, M. (2003). El ácido fórmico como agente de control de Varroa Destructor en Argentina. Buenos Aires, Argentina: Printerline.

Eguaras, M., Cora, D., Sosa, A., \& Ruffinengo, S. (2000). Control de Varroa jacobsoni mediante aplicaciones repetidas de ácido fórmico líquido. Revista Argentina de Producción Animal, 20 (326), 19. 
Espinosa, L., \& Guzmán, E. (2007). Eficacia de dos acaricidas naturales, ácido fórmico y timol, para el control del ácaro Varroa destructor de las abejas (Apis mellifera L.) en Villa Guerrero, Estado de México, México. Veterinaria México, 38 (1), 9-19.

Jordán, B., \& Lalangui, M. (2016). Apicultura en Ecuador. Universidad Técnica de Machala, Ecuador.

Llorete, J., Higes, M., \& Suarez, M. (1996). Tratamientos con productos naturales contra Varroa jacobsoni. Estudio comparativo de varios compuestos. (Timol, Mentol, Alcanfor). Il Congreso de la Sociedad Española de Agricultura Ecológica, Pamplona-Iruña

Milani, N. (1999). The resistance of Varroa jacobsoni Oud. to acaricides. Apidologie, 30, 229-234. DOI: https://doi.org/10.1051/apido:19990211.

Nanetti, A. (2007). Uso de ácido oxálico y otros productos de origen natural para el control de Varroa, Pros y Contras. Revista Agro Sur Chile, 3 (1), 48-50. DOI: https://doi.org/10.4206/agrosur.2007.v35n1-18.

Ruíz, A., Ramírez, E., Maldonado, E., Palafox, J., Ochoa, E., \& López. R. (2012). Incidencia y nivel de infestación por varroasis en abejas (Apis mellifera) en el laboratorio de identificación y diagnóstico apícola de 2002 a 2006. Revista Chapingo Serie Ciencias Forestales y del Ambiente, 18 (2), 175182. DOI: https://doi.org/10.5154/r.rchscfa.2011.03.023.

Ruíz, J. (2012). Control de la varroa con timol. V Jornadas Técnicas de Apicultura de Córdoba, Argentina. Sánchez, C. (2003). Crianza y Producción de Abejas. Buenos Aires, Argentina: Servilibros

Silva, A. (2006). Evaluación del ácido oxálico sobre Varroa destructor Anderson y Trueman (Acari: Mesostigmata), aplicado en otoño sobre colonias de Apis Mellifera L (Hym: Apidae). Apidologie, 62, 145-159.

Stanghellini, R. (2004). Evaluation of selected biopesticides for the late fall control of Varroa mites in a northern temperate climate. American Bee Journal, 144, 475-480. 